\title{
Multi-Topic Multi-Document Summarization
}

\author{
UTIYAMA Masao \\ Communications Research Laboratiory \\ 588-2, Iwaoka, Nishi-ku, Kobe, \\ Hyogo 651-2492, Japan \\ mutiyama@crl.go.jp
}

\author{
HASIDA Kôiti \\ Electrotechnical Laboratory \\ 1-1-4, Umezono, Tukuba, \\ Ibaraki 305-8568, Japan \\ hasida@etl.go.jp
}

\begin{abstract}
Summarization of multiple documents featuring multiple topics is discussed. The example treated here consists of fifty articles about the Peru hostage incident for December 1996 through April 1997. They include a lot of topics such as opening, negotiation, ending, and so on. The method proposed in this paper is based on spreading activation over documents syntactically and semantically annotated with GJ)A (Global Document Annotation) tags. The method extracts important documents and important parts thercin, and creates a network consisting of important cntities and rolations among them. It also identifies cross-document coreferences to replace expressions with more concrete ones. The method is essentially multilingual due to the language-independence of the GDA tagset. This tagset can provide a standard format for the study on the transformation and/or gencration stage of summarization process, among other natural language processing tasks.
\end{abstract}

\section{Introduction}

A large event consists of a number of smaller events. These component events are usually related but such relations may not be strong enough to define larger topics. For example, a war may consist of opening, battles, negotiations, and so on. These relatively independent events are considered to be topics by themselves and would accordingly be reported in multiple news articles.

Summarization of such a large event, or multiple documents about multiple topics, is the concern of this paper. Summarization of multiple documents containing multiple topics is an unexplored rescarch issuc. Some previous studies on summarization (McKeown and Radev,
1995; Barzilay ct al., 1999; Mani and Blocdorn, 1999) deal with multiple documents about a single topic, but not about multiple topics ${ }^{1}$.

In order to summarize multiple documents with multiple topics, one needs a general, semantics-oriented mothod for cvaluating importance. Summarization of a single document may largely exploit the document structure. As an extreme example, the fisst paragraph of a newspaper article often serves as a summary of the entire article. On the other hand, summarization of multiple documents in general must be more based on their semantic structures, because there is no overall consistent document structure across them.

Selection of multiple important topics (not keywords) for multiple-topic summarization has not yet been really addressed in the previous literature. The present paper proposes a method, based on sprearling activation, for $\mathrm{ex}-$ tracting important topics and important documents. Another method proposed which is useful for grasping the overviow of multiple documents is visualization of important entities mentioned and relationships among them. Visualization of relationships among keywords has been studied in the context of information retricval (Niwa et al., 1997; Sanderson and Croft, 1999), but to the authors' knowledge the present study is the first to address such visualization in the context of summarization. Of course a concise summary of the entire set of multiple documents can be obtained by recovering sentences from important entitics and their relationships as demonstrated in section 3.3 .

The present study assumes documents annotated with GDA (Global Document Annota-

\footnotetext{
${ }^{1}$ Maybury (1999) discusses summarization of multiple topies, but in his study the summaries are made from an event database but not from documents.
} 
tion) tags (Hasida, 1997; Nagao and IIasida, 1998). Since the GDA tagset, is designed to be independent of any particular natural language, the proposed method is essentially multilingual. Another merit of using annotated documents is that we can separate the analysis phase from the whole process of summarization so that wo can focus on the latier, generation phase of summarization process. Annotated documents can also be useful for a common input format for the study of summarization, among other natural languago processing tasks.

\section{The GDA Tagset}

GI) $\Lambda$ is a project to make on-line documents machine-understandable on the basis of a linguistic tagset, while developing and sprearing technologies of content-based presentation, retrieval, question-answering, summarization, translation, among others, with much higher quality than before. GD $\Lambda$ thus proposes an integrated global platform for electronic: content authoring, presentation, and reuse. Tho GI) $\Lambda$ tagset $^{2}$ is an XMI, (eXtensible Markup) Janguage) instance which allows machines to automatically infer the semantic and pragmatic structures underlying the raw documents.

Under the current state of the art, GDAtagging is somiautomatic and calls for manual correction by human annotators; otherwise annotation would make no sense. The cost involved here pays, because ammotated documents are generic information contents from which to render diverse types of presentations, potentially involving summarization, narration, visualization, translation, information retrieval, information extraction, and so forth. The present paper concerns summarization only, but the merit of GI)A-tagging is not at all restricted to summarization, and that is why it is considered reasonable to assume GDA-tagged input here.

\subsection{Syntactic structure}

An example of a GD $A$-tagged sentence is shown in Figure 1. <su> means sentential unit. 〈np>, $\langle\mathrm{v}\rangle$, and $\langle\mathrm{adp}\rangle$ stand for noun plirase, verb, and adnominal or adverbial phrase.

<su> and the tags whose name end with ' $p$ ' (such as <adp> and $\langle v p\rangle$ ) are called phrasal tags. In a sentence, an clement (a text span

\footnotetext{
${ }^{2}$ http://ww .etl.go.jp/etl/nl/GDA/tagset.html
}

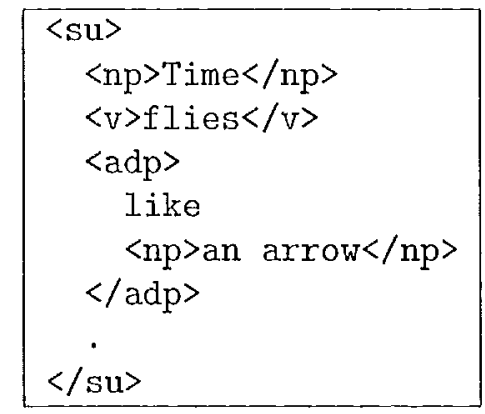

Figure 1: $\Lambda$ (G]) $\Lambda$-tagged sentence.

from a begin tag to the corresponding end tag) is usually a syntactic constituent. The elements enclosed in phrasal tags are called phrasal elements, which cannot be the head of larger elcments. So in Figure 1 'flies' is specified to be the head of the <su> element and 'like' the head of the $\langle a d p\rangle$ element.

\subsection{Coreferences and Anaphora}

Each element may have an identifier as the value for the id attribute. Coreferences, inchuding identity anaphora, aro annotated by tho eq attribute, as follows:

$$
\begin{aligned}
& <\operatorname{lnp} \text { id }=" j 0 ">\text { John }\langle/ \text { np }>\text { beats } \\
& <\text { adp eq="jO" }>\text { his }</ \text { adp }>\text { dog. }
\end{aligned}
$$

When the shared semantic content is not the referent but the type (kind, set, etc.) of the referents, the eq.ab attribute is used like the following:

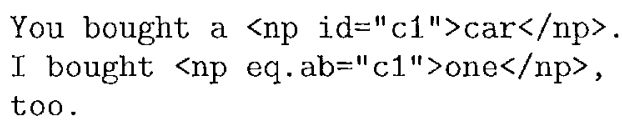

A zero anaphora is encoded as follows:

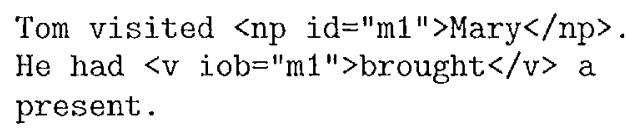

iob $=" m 1 "$ means that the indirect object of brought is clement whose id value is $\mathrm{m} 1$, that is, Mary.

Other relations, such as sub and sup, can also be encoded. sub represents subset, part, or element. $\Lambda$ n example follows:

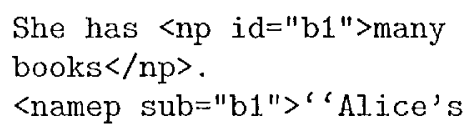


Adventures in Wonderland' '</nameps is her favorite.

sup is the inverse of sub, i.c., includer of any sort, which is superset as to subset, whole as to part, or set as to clement.

Syntactic structures and coreferenees arc essential for the summarization method described in section 3. Further details such as semantics, coordination, scoping, illocutionary act, and so on, are omitted here.

\section{Multi-Document Summarization}

\subsection{Spreading activation}

A set of GDA-tagged documents is regarded as a network in which nodes roughly correspond to GJ)A clements and links represent the syntactic and scmantic relations among them. This network is the tree of GIDA clements plus crossreference (via eq, eq.ab, sub, sup, and so on) links among them. Cross-reference links may encompass different documents. Figure 2 shows a schematic, graphical representation of the network.

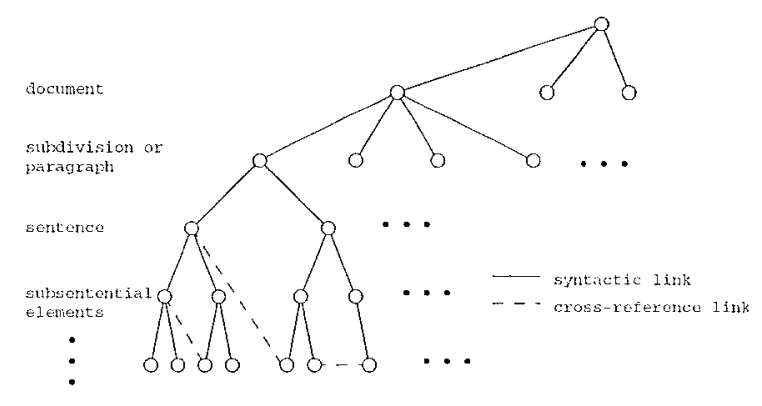

Figure 2: Multi-document network.

Spreading activation is carried out in this network to assess the importance of the elcments. Spreading activation has been applied to summarization of single GDA-tagged documents (Hasida ot al., 1987; Nagao and IIasida, 1998). The main conjecture of the present study is that the merit of spreading activation in that it evaluates importances of semantic entities is greater in summarization of multiple documents with multiple topies, because summarization techniques using document structures do not apply here, as mentioned carlier.

To fit the semantic interpretation, activations spread under the condition that coreferent elements should have the same activation value.
The algorithm ${ }^{3}$ is shown in Figure 3. Here the extcrnal input $c(i)$ to node $i$ represents a priori importance of $i$, which is set on an empirical basis; for instance, an entity ${ }^{4}$ roferrod to in the title of an article tend to be important, and thus $c(i)$ should be relatively large for the corresponding node $i$. The weight $w(i, j)$ of another kind of link from node $i$ to node $j$ may also bo set empirically, but it is fixed to a uniform value in the present work. Let $E(i)$ be the equivalence class of node $i$, that is the set of nodes which are coreferent with $i$ (linked with $i$ via eq relationships). Condition

$$
\sum_{k \in E(i)} \sum_{j \notin E(i)} w(k, j) \leq 1
$$

should be satisfied in order for the spreading activation to converge. This condition is satisfied if we treat each equivalence class of nodes as a virtual node while setting the weights of other types of links to be $\frac{1}{D}$, where $D$ is the maximum degrec of equivalence classes:

$$
D=\max _{i} \sum_{k \in E(i)} \sum_{j \notin E(i)} \delta_{k j}
$$

where $\delta_{k j}$ is 1 if there is a link between node $k$ and node $j$, otherwise it is 0 .

The score score $(i)$ of node $i$ is calculated by summing the activation values of all the nodes under node $i$ in the syntactic tree structure:

$$
\operatorname{scorc}(i)=a(i)+\sum_{j \in \operatorname{ch}(i)} \operatorname{score}(j)
$$

where $a(i)$ is the activation value of node $i$ and $c h(i)$ is the set of child nodes of node $i$. ch $(i)$ is cmpty if node $i$ is a leaf node, or a word. This score is regarded as the importance of node $i$.

\subsection{Extraction of important documents and sentences}

Extraction of important documents is simple once the scores of the nodes in the network are obtained. Sorting the document nodes according to their scores and extracting higher-ranked ones is sufficient for the purpose.

\footnotetext{
${ }^{3}$ Another spreading activation algorithm is discussed by Mani and Bloedorn (1999). The comparison is a f11ture work.

'We use the terms 'entity', 'node', and 'element' intorchangeably.
} 


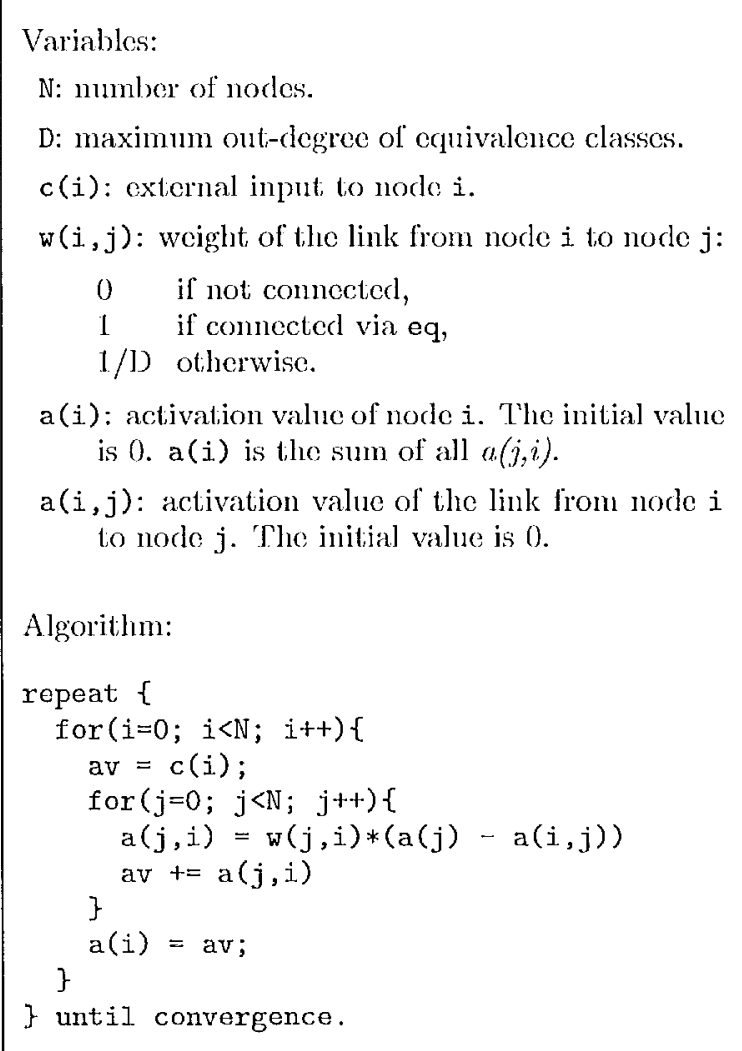

Figure 3: Spreading activation algorithm.

Similas procedure is used to extract important sentences from an important document. Extracted sentences aro pruned according to their syntactic structures. Anaphoric expressions such as he or she are substituted by their antecedents if necessary.

An experiment has been conducted to test the effectiveness of the proposed algorithm. The example set contains fifty Japanese articles about the Peru hostage incident which continued over four months from December 1996 to April 1997. They include a lot of topics such as opening, negotiation, settlement, and so on. The GI $\Lambda$ tagging of these articles has involved automatic morphological analysis by JUMAN (Kurohashi and Nagao, 1998), automatic syntactic analysis by KNP' (Kurohashi, 1998), and manual anmotation encompassing morphology, syntax, coreference, and anaphora. The types of anaphora identified here are mainly plain coreference and zero anaphora. Cross-document corcferences among entities have been automatically identi- fied by exact string matching. ${ }^{5}$ They contained crrors but those errors were not corrected for the experiment. Cross-document coreferences found were 'P'Pru'(49), 'Japan'(39), 'Pcru President' (15), 'members of 'Tupac $\Lambda$ maru'(9), ... and so on, where the numbers indicate the numbers of documents which contain these expressions.

The external inputs to nodes have been defined according to the corresponding nodes: $c(i)=10$ if node $i$ 's antecedent dominates sentences (c.g., a node corcferring with a paragraph). This sets a preforence for nodes which summarize preceding sentences. $c(i)=5$ if node $i$ is in the title of an article, because at title is usually important. Otherwise $c(i)=1$. These crude parameter values have been set by the authors on the basis of the investigation of summarizations of various documents.

Two important topies, the opening (first attack by Tupac $\Lambda$ maru) and the settlement (attack by the Peruvian government comman(los), have been extracted from the four highest ranked articles, cven though temporal information has not been incorporated in the algorithm. The opening article is the first article of the sample document set. However, the settement article is the sixth last one. So more extraction of the last article would miss the settement.

The 25\% summaries of the two articles made by extracting and pruning sentences are shown bolow together with their English translations:

日本大使公畉に武装午リラ、パーティーに乱入 鋴慗、200人が人筫——ペルー

甘本、ペルーの两国関係者多数が人筫にとられ た。武壮グループは約 20 人で、うち複数が公勋

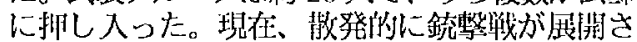
れているという。

Armed gucrillas broke into a party at Japanese ambassador's residence. Gunshots. 200 helk in hostage. P'eru.

Many pcople from Japanese and Peruvian sides were held in hostage. The armed group consists of abont twenty people, several of which broke into the ambassador's residence. It is reported that there are intermitient shootings now.

and

\footnotetext{
${ }^{5}$ We are planning to incorporate recent results (Bagga and Baldwin, 1998) to identify cross-document coreferences.
} 


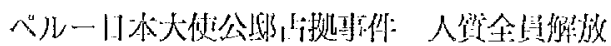

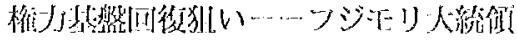

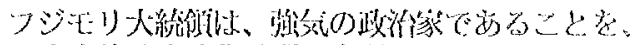

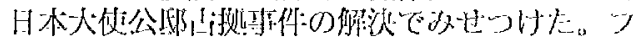

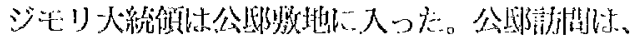

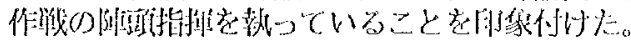

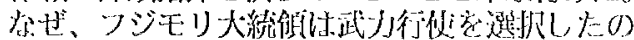

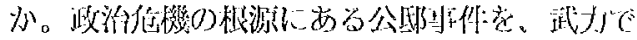

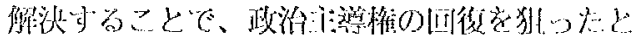
W之心。

Japanese anbassador's residence possecsion] incident in P'oru. All hostages released. Aim at rocovering his power basis P'resident linjimori.

President Fujimori demonstrated himsell as a strong politician by rewolving the Japaness anbassarlor's residence possession incident. Ito ontered the rosideneo site. 'T'his visit to the residence inpressed that lo was leading" the operation himself. Why did he choose to resort to amos? Wo can say that he anined at recovering his political teadesthip by re. solving through mititary power the residence incident, which is at the root of the political crisis:

\section{3..8 Mabily-relation graph}

The score seore $(i, j)$ of a relation betwecen two entitios $i$ and $j$, is defined by:

$$
\begin{aligned}
& \text { score }(i, j) \\
& |H(i)| a(i)+|k(j)| a(j) \\
& +\sum_{s \in S(\omega(i)) \cap S(s(j))}^{+s c o t e}(s)
\end{aligned}
$$

where $S(f(i))$ is the set of sentence nodes which dominate one of the nodes in $l e(i)$ and $\mid$ teiti $\mid$ is the number of nodes in $E(i)$. IS(i), $a(i)$, and score $(s)$ have been defined in Section 3.1. $|E(i)| a(i)$ is an analogy of ' $t f \times$ idf', which is in measiure of term importance widely used in information rotricval.

If $\operatorname{seore}(i, j)$ is sufficiently large, then $S(L(i)) \cap S(E(j))$ (the sentences containing both the entities) can constitute a crossdocument summary concorning $i$ and $j^{6}$.

An culity-rolation graph (L-R graph) is made. of the relations highly ranked in terms of the score defined in (2). Figure 4 shows the ti-R graph made of the top eleven relations extracted from the articles about Peru hostage incident. The numbers near the lines represent the ranks of the relations.

\footnotetext{
${ }^{6}$ Coroference chains are used to summarize singlo doc uments by Azzam et al. (1999).
}

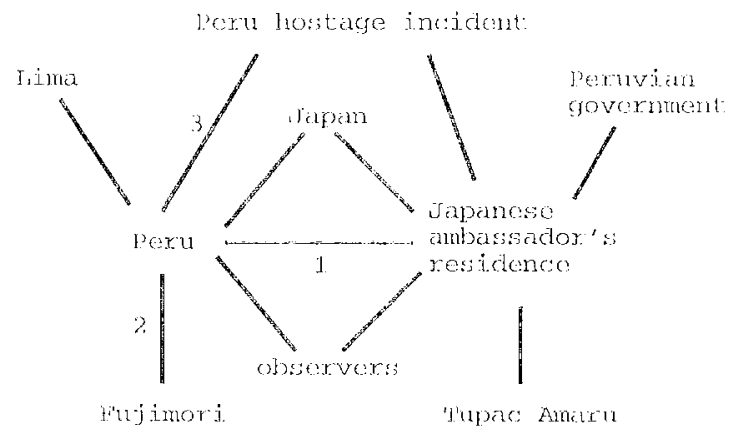

Jigure A: fi R graph of Peru hostage incident.

The topmanked relation was the one be tween P'om and Anpanese ambassador's residence. Three sentences extracted from the cight sentences which costialned both of the entitios were as follows." They were listed in chrono logical onder which was identified by the dato information in the artickes.

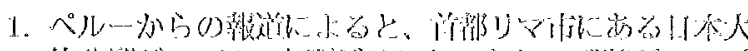

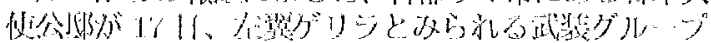

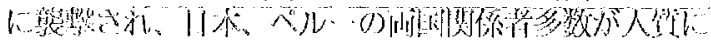
と水\%。

According to reports from l'ern, an the thth the Japanese andatsinderes residence in lima, the capp tall, was at iacked by an armed gromps, allowedly Ieftist goerrillase and many people frout both Japanese and Peruvian sicles were theld in hostage.

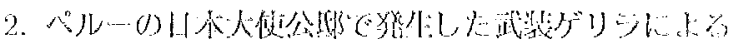

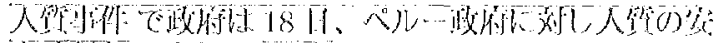

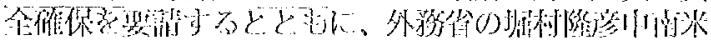

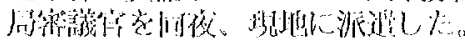

Concerning the hostage incidont at the Japenese nombassador's residence caused by armed gratrilla, on the 18 th the government secpested the Peruvian grovernment to assime the safedy of the hostages, and sont Mr. Holidet lakalniko, coordinator, Division of Midalo and South Amorica. . .

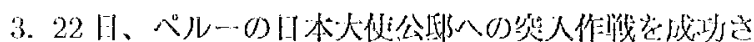

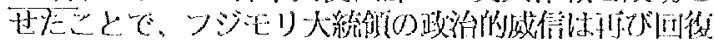
に向小うるとになるた。

President lujimori's political authomity will recoven because he succeded in the operation to break into the Japanese ambassialor's residence in Peru on the g2nd.

These sentences, extracted from different atticles, have been paraphrased on the basis of

\footnotetext{
${ }^{7}$ T'liese sentences wore selected manually to demonstrate the possibility of cross document sunmarization based on corelerence.
} 


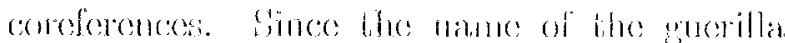

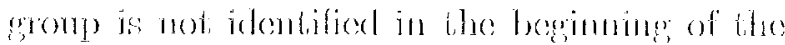
incident, the oxpresison "y

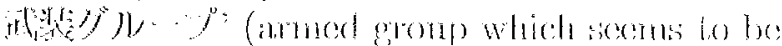

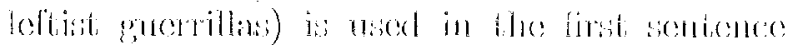

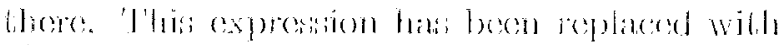

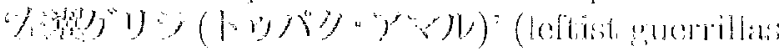

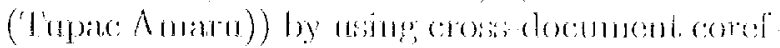

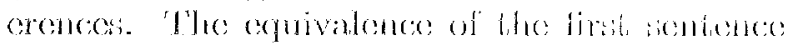

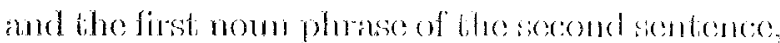

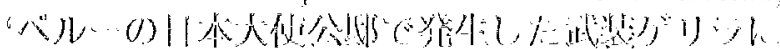

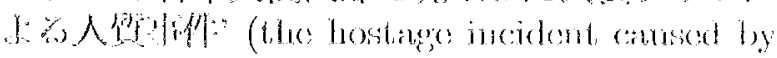

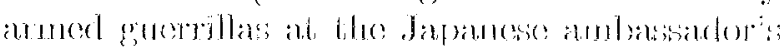

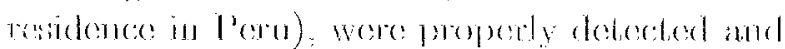

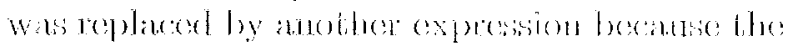

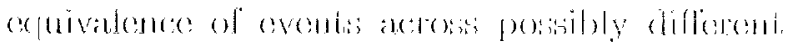

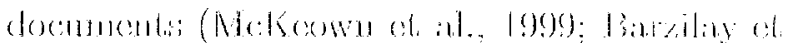

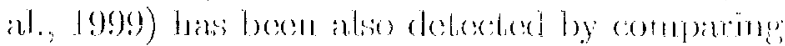

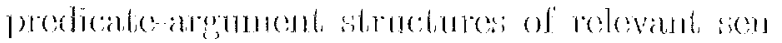

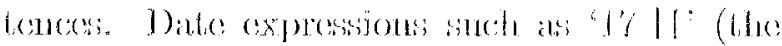

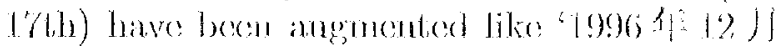

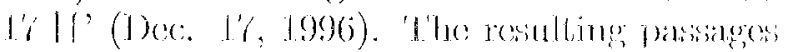

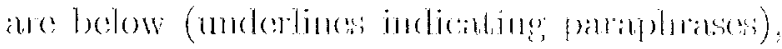

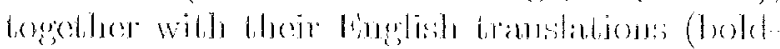

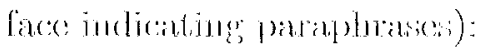

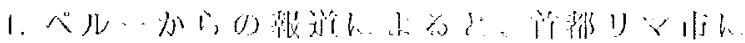
汸\%

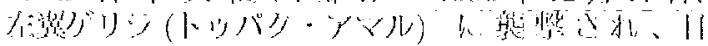

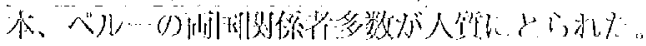

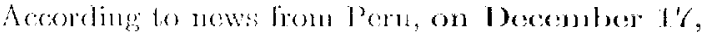
1996 the dapanese ambatwator so residenes in tima, the capital, was attateked loy leftiot pherrillats (Tiume: Amaru), and many people from both Japanese and l'ernvian sides were hetd in heritiege.

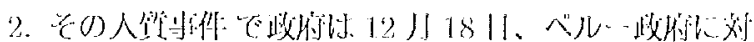

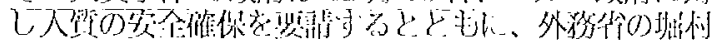

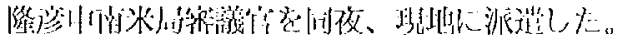

Concerning the hostage iucident, the: fowernmont requested the Peruviau govermuent to atsiure the hostages' salety on Decomber the 18th, and sent, Mr. IIORIU'T 'Lakahiko, coordinater, Division of Middle and South Auerica, Ministry of lntermational Afians, to l'eru on that nighth. $\cdots$

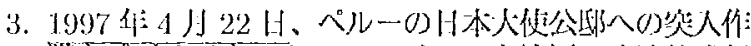

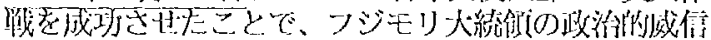

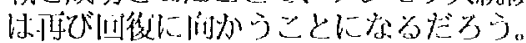

President linjinori's political authority will rocover because he succeded in the operation to loreak into the fapanese ambassador's residenes in l'eru on $\Lambda$ pril 22,1997 .

\section{Discosision}

\section{A.1 Wvaluation}

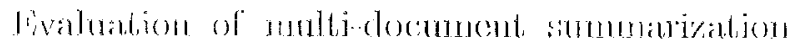

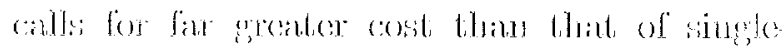

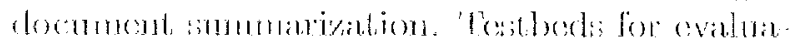

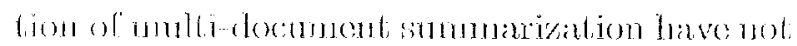

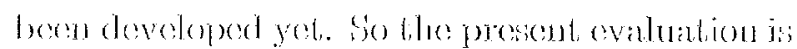
bimbet he the sample sed of articles mentioned

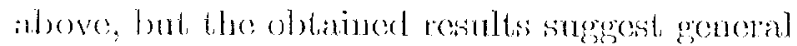

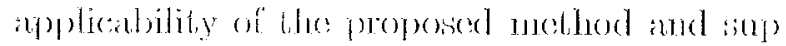

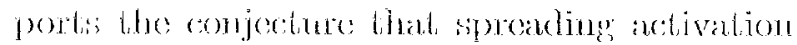

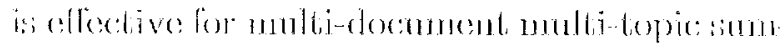
matration.

As discussed in the previons section, the pro

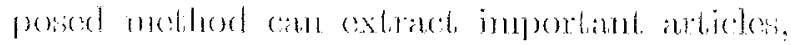

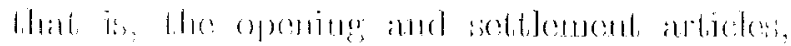

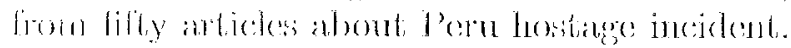

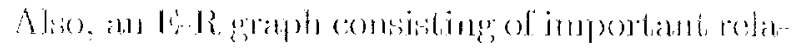

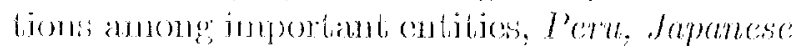
ambassador:" residenes, 'Tupat Amante, and so

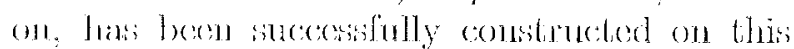

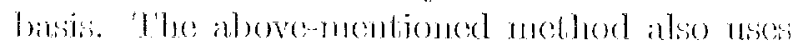

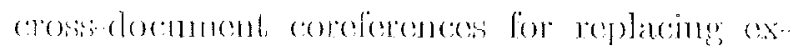

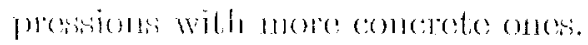

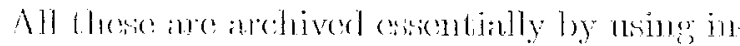

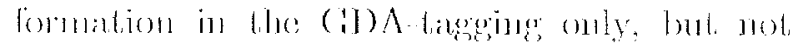

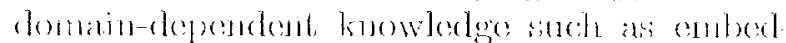
ded in lomplates for infommation ostmations.

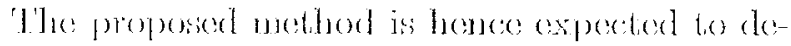

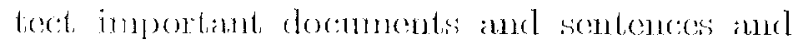

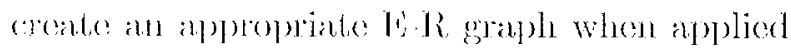

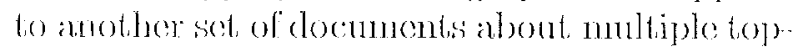
ics.

\subsection{Tramsformalion}

I'lue processis of smmmarization cam be decomposed inbo three stiagos (Sparck Jonos, 1999):

1. source toxt inderpletation to source text reprosontation,

2. somec representation transformation to summary text representition, and

3. summary text generution from summary reprosontation.

(iD) $\Lambda$-lagged documontis are rogarded as somreo toxt representations. The nothod clescribed above focuses on the transformation stage. Its; multi-linguality cones from the multi-linguality of tiho stiage. 


\section{Conclusion}

Summarization of multiple documents about multiple topics has been discussed in this paper. The method proposed here uses spreading activation over documents syntactically and semantically annotated with GD $\Lambda$ tags. It is capable of:

- cxtraction of the opening and settlement articles from fifty articles about a hostage incident,

- creation of an entity-relation graph of important relations among important entities,

- extraction and pruning of important sentences, and

- substitution of expressions with more concrete ones using cross-document coreferences.

The method is essentially multilingual because it is based on GDA tags and the GDA tagset is designed to address multilingual coverage. Since this tagset can embed various linguistic information into documents, it could be a standard format for the study of the transformation and/or generation stage of document summarization, among other natural language processing tasks.

\section{References}

Saliha Azzam, Kevin IIumphreys, and Robert Gaizauskas. 1999. Using coreference chains for text summarization. In ACI'99 Workshop on Coreference and Its Applications, pages $77-84$.

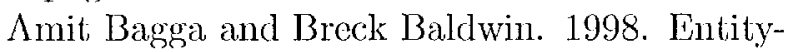
based cross-document coreferencing using the vector space model. In COLING-ACL'98, pages 79-85.

Regina Barzilay, Kathleen R. McKeown, and Michael Elhadad. 1999. Information fusion in the context of multi-document summarization. In $A C L^{\prime} 99$, pages 550-557.

Kôiti Hasida, Syun Ishizaki, and Hitoshi Isahara. 1987. A connectionist approach to the generation of abstracts. In Gerard Kempen, editor, Natural Langauge Generation: New Results in Artificial Intelligence, Psychology, and Linguistics, pages 149-156. Martinus $\mathrm{Ni}-$ jhoff.
Kôiti Hasida. 1997. Global Document Annotation. In NLPRS'9\%, pages 505-508.

Sadao Kurohashi and Makoto Nagao. 1998. Japanese morphological analysis system JUM $\Lambda \mathrm{N}$ manual.

Sadao Kurohashi. 1998. Japanese syntactic analysis system KNP manual.

Inderjeet Mani and Eric Bloedorn. 1999. Summarizing similarities and differences among related documents. In Inderjoet Mani and Mark T. Maybury, editors, ADVANCES IN AUTOMATIC TEXT SUMMARIZATION, chapter 23, pages 357-379. The MIT Press.

Mark T. Maybury. 1999. Generating summaries from event data. In Inderjeet Mani and Mark T. Maybury, editors, ADVANCES IN AUTOMATIC TEXT SUMMARIZATION, chapter 17 , pages $265-281$. The MIT Press.

Kathleen McKcown and Dragomir R. Radev. 1995. Generating summaries of multiple news articles. In SIGIR'95, pages 74-82.

Kathleen R. McKeown, Judith L. Klavans, Vasileios Hatzivassiloglou, Regina Barzilay, and Elezar Eskin. 1999. Towards multidocument summarization by reformulation: Progress and prospects. In $\Lambda A A I-99$, pages 453-460.

Katashi Nagao and Kôiti Hasida. 1998. Automatic Text Summarization Based on the Global Document Annotation. In COLINGACL'98, pages 917-921.

Yoshiki Niwa, Shingo Nishioka, Makoto Iwayama, Akihiko Takano, and Yosihiko Nitta. 1997. Topic graph generation for query naviagation: Use of frequency classes for topic extraction. In NLPRS'g7, pages 95-100.

Mark Sanderson and Bruce Croft. 1999. Deriving concept hierarchies from text. In SIGIR'99, pages 206-213.

Karen Sparck Jones. 1999. Automatic summarizing: factors and directions. In Inderjeet Mani and Mark T. Maybury, editors, $A D$ VANCES IN A UTOMATIC TEXT SUMMARIZATION, chapter 1, pages $1-12$. The MIT Press. 